\title{
Method for instrumentation of orthotic joints for measurement of internal joint loads
}

DOI 10.1515/cdbme-2017-0008

\begin{abstract}
Introduction: The lack of knowledge of mechanical loads in orthotic joints can lead to oversized or breaking components. Previous studies suffer from small sample size and technical limitations. The goal of this study was to develop and validate a method that allows the direct measurement of moments in sagittal, frontal and transverse planes in knee and ankle joints of existing custom made orthoses.

Methods: We developed a modular measurement system based on standard joint components, which were instrumented with strain gauges. To ensure sufficient signals and reduce cross talk an iterative approach based on FEM simulation was utilized. The system also contains inertial sensors for mobile gait analysis.

Results: Instrumented joints show good results regarding linearity, hysteresis and cross talk. First pilot trials with post-polio and ICP patients demonstrated that joint loads depend on several factors and not solely on body weight. If combined with conventional gait analysis, measurement results can characterize the individual muscle situation of the patient.

Conclusion: A novel method for obtaining data on loads in orthotic components was developed and validated. It provides the basis to develop safety testing standards and clinical guidelines, as well as allowing individual optimization of orthotic devices.
\end{abstract}

Keywords: AFO; gait analysis; joint loads; KAFO; orthotic devices.

\section{Introduction}

Size, bulkiness and weight are main reasons for dissatisfaction with orthotic devices [1]. However, the optimization of

\footnotetext{
*Corresponding author: David Hochmann, Münster University of Applied Sciences, Steinfurt, Germany, E-mail: david.hochmann@fhmuenster.de

Lucien Opitz: Münster University of Applied Sciences, Steinfurt, Germany, E-mail: lucien.opitz@fh-muenster.de
}

orthotic components as joints and bars is still very difficult, due to lack of established design standards. Moreover, there is a limited knowledge of possible loads in orthotic components and how they depend on individual patient characteristics as limb misalignments and remaining muscle function.

In the past, attempts were made to determine the loads in orthotic joints using conventional gait analysis [2] or industrial force sensors [3, 4]. However, gait analysis studies completely neglect the contribution of remaining muscles. Industrial sensors were used as a replacement of orthotic joints, which highly influenced the gait pattern and only allowed the measurement in locked condition. Moreover, load sensor and fixtures added up to $70 \%$ more weight to the orthosis [4]. The first trial to overcome these limitations used small instrumented adapters placed between joints and bars, which only measured sagittal moments but could be used in unlocked condition and at every joint position, including ankle [5]. Unfortunately, due to additional height of instrumented adapters, they cannot be integrated into existing orthoses. For each patient included in the study, a new custom orthosis needs to be manufactured, leading to high costs and limited sample size.

To successfully establish a data base allowing to analyze the impact of patient characteristics on loads, a measurement system which is compatible with existing orthoses is needed. It is also important to be able to measure the moments in frontal and transverse planes due to their impact on mechanical safety.

\section{Methods}

To achieve that, we developed a measurement method that uses common orthotic joints as deformation element for instrumentation with strain gauges, as instrumented joints can easily replace build-in joints of the same type in existing orthoses. However, because the original design of the joint cannot be changed, we had to address two major issues. First, it was difficult to obtain sufficient signals due to high stiffness of the joint body. Second, the asymmetrical form 


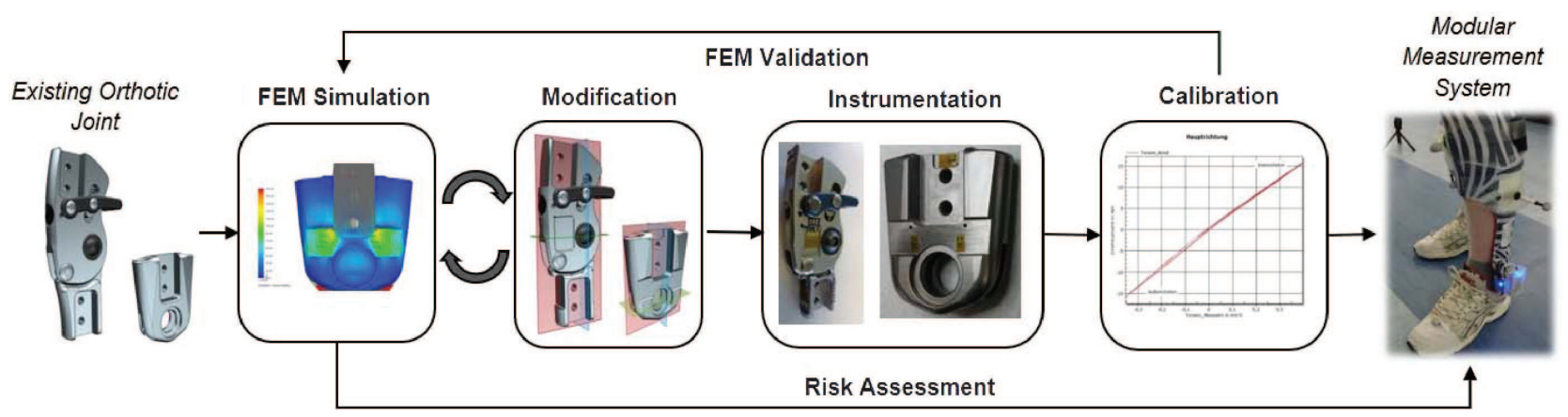

Figure 1: Method for instrumentation of orthotic joints with strain gauges.

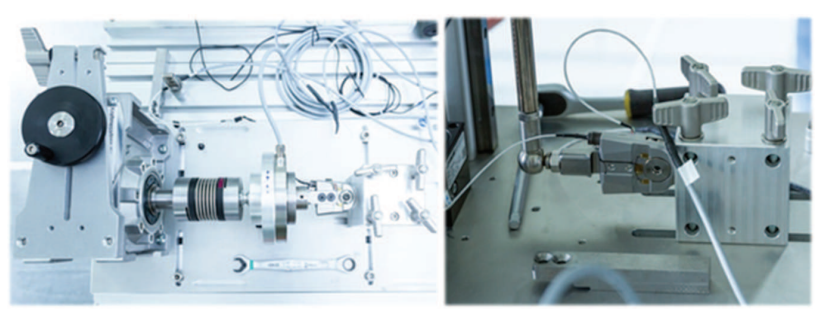

Figure 2: Custom build calibration devices.

of the joint led to high cross talk between sagittal moment and moments measured in other planes.

The general method to overcome these challenges is shown in Figure 1. We used finite element method (FEM) to calculate local deformations of the joint body and thus find the optimal spots for strain gauge placement. As the deformation of original joint was still not sufficient, we tested a number of design modification concepts using FEM simulation. The goal of these iterative steps was to achieve sufficient signals without affecting the mechanical safety of the joint. After the optimum modification scenario was found, the joint was mechanically modified and instrumented with strain gauges. The instrumented joint was calibrated using custom build calibration devices (Figure 2) and characterized regarding linearity, hysteresis and cross talk. The resulting calibration curve was also used to ensure that FEM simulation was valid.

Instrumented joints are connected to custom build wireless electronics for amplifying and logging. Calibration data for all planes is stored directly on chip. Our system also includes inertial sensors for mobile measurement of kinematic gait parameters and a mobile photoelectric trigger for synchronization with conventional gait analysis.

Before the instrumented joints could be used for measurement, we had to assess the risk due to modification of original design. For that, we calculated deformations of critical parts of the joint using previously validated FEM. For the maximum load level provided by the manufacturer all deformations were in acceptable range.

\section{Results}

In the use, it takes only a few minutes to replace buildin joints with our new measurement system. The weight increase for the orthosis is very small (60 g per joint). As the system is wireless, there are no cables that interfere with moving parts of the orthosis.

Until now two pilot trials using the new measurement system were performed. In the first one we measured the moments in an orthotic ankle joint of an AFO in combination with conventional gait analysis using VICON. The measurements were performed with a healthy volunteer (female, 32 years old, $55 \mathrm{~kg}, 168 \mathrm{~cm}$ ) wearing a stiff unilateral GRAFO often used for ICP patients (Figure 3A) for different activities (level walking, stair and ramp climbing, simulated crouch gait etc.).

As expected, the highest moments for all activities were measured in the sagittal plane. For example, for level walking using a $0^{\circ}$ degree dorsiflexion stop, maximum moments were $53.6 \pm 1 \mathrm{Nm}$ in the sagittal plane, $10.7 \pm 1.5 \mathrm{Nm}$ in the frontal plane and $10.3 \pm 1.5 \mathrm{Nm}$ in the transverse plane.

As an example, the normalized plots of sagittal ankle moment measurements for level walking with both systems are shown in Figure3C. Both curves are very similar in their pattern. However, the magnitude of total external moment measured by VICON is much higher than the moment in orthotic joint. That means that most of the external moment is compensated by internal muscle moment of the healthy volunteer. Thus, the difference between total external moment measured by VICON and orthotic joint moment measured by our system can be used to characterize the remaining muscle function of the individual patient.

We also used the trial to compare the built-in mobile gait analysis to VICON as gold standard. For all activities, the differences in segment angles and time-distance parameters between VICON and our system were very small $(<3 \%)$. 

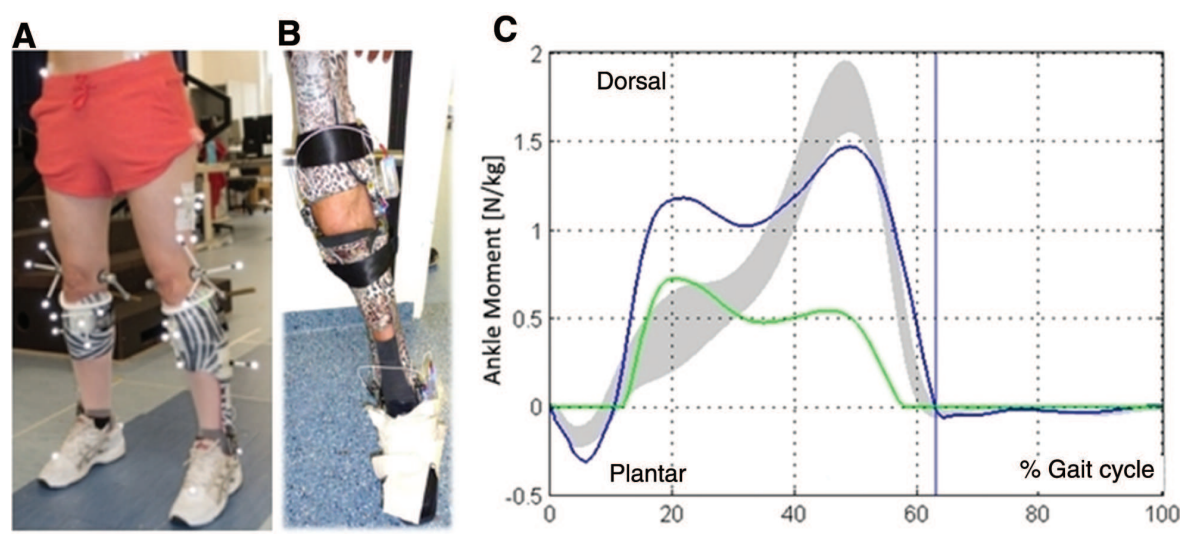

Figure 3: (A) Instrumented GRAFO (B) instrumented KAFO (C) results of combined sagittal ankle moment measurement (blue line -VICON moment, green line - orthotic joint moment, grey - physiological range).

Table 1: Peak loads in KAFO Joints for level walking.

\begin{tabular}{lrrl}
\hline Joint position & $\begin{array}{r}\text { Sagittal } \\
\text { moment [Nm] }\end{array}$ & $\begin{array}{r}\text { Frontal } \\
\text { moment [Nm] }\end{array}$ & $\begin{array}{l}\text { Transverse } \\
\text { moment [Nm] }\end{array}$ \\
\hline Knee medial & 25.2 & 2.8 & 3.4 \\
Knee lateral & 22.8 & 5.8 & 3.3 \\
Ankle medial & 35.2 & 4 & n.a. \\
Ankle lateral & 23.4 & 3.1 & 3.9 \\
\hline
\end{tabular}

The second trial was done in cooperation with local orthopedic workshop. We replaced all ankle and knee joints in an existing KAFO of a 55-year-old polio survivor (Figure 3B) in order to measure the peak loads in orthotic joint during different activities. The results for level walking are shown in Table 1.

The measured sagittal moments were then compared to moments used by manufacturer during durability testing to find the optimal bar thickness in order to counteract oversizing. Furthermore, moments in frontal and transverse planes were used to evaluate the safety of unilateral KAFOdesign. The results also confirmed previous findings that joint loads depend on several factors (mostly remaining muscle function and limb misalignments) and not solely on body weight.

\section{Discussion and outlook}

Originally, the primary goal of our research project was to measure the loads in orthotic joint structures in order to develop validated design and safety testing standards for orthotic components. However, our preliminary results show that the suggested method of measurement can be also used to:

- control electronic components as SCO and SSCO joints,
- characterize the individual muscular situation of the patient (in combination with conventional gait analysis),

- adjust the design of the orthosis to individual needs of the patient,

- monitor the functional changes due to successful rehabilitation or progressive illness.

The results show, that it is possible to convert existing orthotic joints into a modular measurement system that does not suffer from limitations of previous studies as high additional weight and changes in gait patterns [3, 4]. Moreover, the measurements can be performed not only in the gait lab, but also in the usual environment of the patient.

The modern methods of muscle function assessment used by orthopaedic technicians in the practice, such as Janda Scale, suffer from very poor repeatability. The shown method of assessment of remaining muscle function with a combination of our system with VICON gait analysis can provide a better alternative. However, we have yet to prove its suitability for daily use and to compare it with other existing methods like mechanomyography.

Up to date, it is also not possible to reliably measure pathologic internal moments in case of spasticity. Clinically used descriptive scales, such as Modified Ashworth Scale (MAS), lack objectivity and can only be used as outcome measures. Even EMG signals are not sufficiently sensitive to detect the dynamic changes in tonus of a spastic muscle. We hypothesize that the tonus changes will result in a change of the load pattern in orthotic components, which can be directly measured with our system.

In the future, after the proposed method is used on a larger and more diverse group of patient there should 
be a sufficient data not only to develop design and safety testing standards for KAFOs but also to establish clinical guidelines for patient device selection.

Acknowledgment: Special thanks to Heidelberg Motion Lab and UKM Protec.

\section{Author's Statement}

Research funding: The research project was partially supported by Ottobock Healthcare (Duderstadt, Germany). The sponsor was not involved into collection, analysis or interpretation of data. Conflict of interest: David Hochmann has consultancy agreement with the sponsor. Informed consent: Informed consent has been obtained from all individuals included in this study. Ethical approval: The research related to human use complies with all the relevant national regulations, institutional policies and was performed in accordance with the tenets of the Helsinki Declaration. An approval by an' institutional review board was not needed.

\section{References}

[1] Batavia AJ, Hammer G. Toward the development of consumerbased criteria for the evaluation of assistive devices. J Rehabil Res Dev. 1990;27:425-36.

[2] Johnson GR, Ferrarin M, Harrington M, Hermens H, Jonkers I, Mak $P$, et al. Performance specification for lower limb orthotic devices. Clin Biomech. 2004;19:711.

[3] Andrysek J, Redekop S, Matsui NC, Kooy J, Hubbard S. A method to measure the accuracy of loads in knee-ankle-foot orthoses using conventional gait analysis. Arch Phys Med. 2008;89:1372-9.

[4] Bernhardt K, Kaufman K. Loads on the uprights of a knee-ankle-foot orthosis. Prosthet Orthot Int. 2011;35:106-12.

[5] Hochmann D, Schmalz T. Direct measurement of loads in KAFO joints for locked and sco conditions. AOPA Annual Meeting, Boston; 2012. 